\title{
Solving a Fully Fuzzy Linear Programming Problem through Compromise Programming
}

\author{
Haifang Cheng, ${ }^{1}$ Weilai Huang, ${ }^{1}$ and Jianhu Cai ${ }^{2}$ \\ ${ }^{1}$ School of Management, Huazhong University of Science and Technology, Wuhan 430074, China \\ ${ }^{2}$ College of Economics and Management, Zhejiang University of Technology, Hangzhou 310023, China \\ Correspondence should be addressed to Jianhu Cai; hzdcjh@yahoo.com
}

Received 22 February 2013; Accepted 9 May 2013

Academic Editor: Reinaldo Martinez Palhares

Copyright (C) 2013 Haifang Cheng et al. This is an open access article distributed under the Creative Commons Attribution License, which permits unrestricted use, distribution, and reproduction in any medium, provided the original work is properly cited.

\begin{abstract}
In the current literatures, there are several models of fully fuzzy linear programming (FFLP) problems where all the parameters and variables were fuzzy numbers but the constraints were crisp equality or inequality. In this paper, an FFLP problem with fuzzy equality constraints is discussed, and a method for solving this FFLP problem is also proposed. We first transform the fuzzy equality constraints into the crisp inequality ones using the measure of the similarity, which is interpreted as the feasibility degree of constrains, and then transform the fuzzy objective into two crisp objectives by considering expected value and uncertainty of fuzzy objective. Since the feasibility degree of constrains is in conflict with the optimal value of objective function, we finally construct an auxiliary three-objective linear programming problem, which is solved through a compromise programming approach, to solve the initial FFLP problem. To illustrate the proposed method, two numerical examples are solved.
\end{abstract}

\section{Introduction}

Linear programming (LP) has important applications in many areas of engineering and management. In these applications, since the real-world problems are very complex, the parameters of LP are usually represented by fuzzy numbers. Therefore, many researchers have shown interest in the area of fuzzy linear programming (FLP).

Recently fuzzy set theory has been applied in many research regions, since fuzzy set theory is effective to solve the decision-making problems with imprecise data [1-3]. Several kinds of the FLP problems have appeared in the literature [4-16]. Delgado et al. [4] have proposed a general model for the FLP problems in which constraints are fuzzy inequality and the parameters of constraints are fuzzy numbers but the parameters of the objective function are crisp. Rommelfanger [5] has also proposed a general model for the FLP problems and the main difference compared with [4] is that here parameters of the objective function are also fuzzy numbers. Considering the different hypotheses, researchers [6-13] have proposed some particular FLP problems, which can be deduced from the general model. In order to solve these FLP problems, different approaches have been proposed too. Some methods are based on the concepts of the superiority and inferiority of fuzzy numbers [7], the degrees of feasibility [8], the satisfaction degree of the constraints [10], and the statistical confidence interval [11]. Other kinds of methods are multiobjective optimization method [6], penalty method [12], and semi-infinite programming method [13]. Similar other interesting works also can be found in the literature [14-16]. Mahdavi-Amiri and Nasseri [14] develop a new dual algorithm for solving the FLP problem directly. Ganesan and Veeramani [15] propose a method for solving fuzzy linear programming problems without converting them to crisp linear programming problems. Maleki et al. [16] propose a good method for solving an FLP problem, and an auxiliary problem is introduced in their model.

In recent years, several kinds of the fully fuzzy linear programming (FFLP) problems in which all the parameters and variables are represented by fuzzy numbers have appeared in the literature [17-21]. Some authors [17, 18] have discussed FFLP problems with crisp inequality constraints 
and obviously different methods for solving them have been proposed. In these methods the fuzzy optimal solutions of the FFLP problems are obtained by converting FFLP problem into crisp linear programming (CLP) problem. Other authors $[19,20]$ have discussed FFLP problems with crisp equality constraints and different methods for solving them have been proposed too. In their methods, the method proposed by Lotfi et al. [19] can only obtain the approximate solution of the FFLP problems, but the method proposed by Kumar et al. [20] can find the fuzzy optimal solution which satisfies the constraints exactly. Guo and Shang [21] propose the computing model to the positive fully fuzzy linear matrix equation, and the fuzzy approximate solution is obtained by using pseudoinverse. However, in most of previous literatures, all constraints of FFLP problems have the crisp form.

In this paper, an FFLP problem with fuzzy equality constraints has been considered. In order to solve it, we first transform the FFLP problem into a crisp three-objective LP model by considering expected value and uncertainty of fuzzy objective and the feasibility degree of fuzzy constrains. Then we solve it using a compromise programming (CP) approach.

This paper is organized as follows. In Section 2 some basic definitions, arithmetic operations, and comparison operations between two triangular fuzzy numbers are reviewed. In Section 3 the processes for transforming the FFLP problem with fuzzy equality constraints into crisp problem are described. In Section 4 a crisp three-objective LP model to find the fuzzy optimal solution of the FFLP problem is built, and the model is solved through CP in Section 5. In Section 6 a numerical example is given. Conclusions are discussed in Section 7.

\section{Preliminaries}

2.1. Basic Definitions. In this paper, the triangular fuzzy numbers are considered because this form of fuzzy numbers is very simple and popular. Moreover, we can express and estimate many other types of fuzzy numbers with triangular fuzzy number [7]. The triangular fuzzy numbers are defined as follows.

Definition 1 (see $[20,22])$. A fuzzy number $\widetilde{A}=\left(a^{(1)}, a^{(2)}\right.$, $\left.a^{(3)}\right)$ is said to be a triangular fuzzy number if its membership function is given by

$$
\mu_{\widetilde{A}}(x)= \begin{cases}\frac{x-a^{(1)}}{a^{(2)}-a^{(1)}}, & a^{(1)} \leq x \leq a^{(2)}, \\ \frac{x-a^{(3)}}{a^{(2)}-a^{(3)}}, & a^{(2)} \leq x \leq a^{(3)}, \\ 0, & \text { otherwise. }\end{cases}
$$

Definition 2 (see $[20,22])$. A triangular fuzzy number $\left(a^{(1)}\right.$, $\left.a^{(2)}, a^{(3)}\right)$ is said to be nonnegative fuzzy number if and only if $a^{(1)} \geq 0$.
Definition 3 (see [23]). Let $\widetilde{A}=\left(a^{(1)}, a^{(2)}, a^{(3)}\right)$ and $\widetilde{B}=\left(b^{(1)}\right.$, $b^{(2)}, b^{(3)}$ ) be two triangular fuzzy numbers, and then the similarity between $\widetilde{A}$ and $\widetilde{B}$ can be defined as

$$
\begin{aligned}
S(\widetilde{A}, \widetilde{B})= & 1-\frac{1}{4 u} \\
& \times\left(\left|a^{(1)}-b^{(1)}\right|+2\left|a^{(2)}-b^{(2)}\right|+\left|a^{(3)}-b^{(3)}\right|\right),
\end{aligned}
$$

where $u=\max \left(a^{(3)}, b^{(3)}\right)-\min \left(a^{(1)}, b^{(1)}\right)$.

Definition 4 (see $[20,24]$ ). A ranking function is a function $\mathfrak{R}: F(R) \rightarrow R$, where $F(R)$ is a set of fuzzy numbers defined on set of real numbers, which maps each fuzzy number into the real line, where a natural order exists. Let $\widetilde{A}=$ $\left(a^{(1)}, a^{(2)}, a^{(3)}\right)$ be a triangular fuzzy number; then

$$
\Re(\widetilde{A})=\frac{a^{(1)}+2 a^{(2)}+a^{(3)}}{4} .
$$

2.2. Arithmetic Operations. In the following, arithmetic operations between two triangular fuzzy numbers, defined on universal set of real numbers $R$, are reviewed [20,22].

Let $\widetilde{A}=\left(a^{(1)}, a^{(2)}, a^{(3)}\right)$ and $\widetilde{B}=\left(b^{(1)}, b^{(2)}, b^{(3)}\right)$ be two triangular fuzzy numbers; then

(i) $\widetilde{A} \oplus \widetilde{B}=\left(a^{(1)}, a^{(2)}, a^{(3)}\right) \oplus\left(b^{(1)}, b^{(2)}, b^{(3)}\right)=\left(a^{(1)}+b^{(1)}\right.$, $\left.a^{(2)}+b^{(2)}, a^{(3)}+b^{(3)}\right)$,

(ii) $-\widetilde{A}=-\left(a^{(1)}, a^{(2)}, a^{(3)}\right)=\left(-a^{(3)},-a^{(2)},-a^{(1)}\right)$,

(iii) $\widetilde{A} \odot \widetilde{B}=\left(a^{(1)}, a^{(2)}, a^{(3)}\right) \odot\left(b^{(1)}, b^{(2)}, b^{(3)}\right)=\left(a^{(1)}-b^{(3)}\right.$, $\left.a^{(2)}-b^{(2)}, a^{(3)}-b^{(1)}\right)$,

(iv) let $\widetilde{B}=\left(b^{(1)}, b^{(2)}, b^{(3)}\right)$ be a nonnegative triangular fuzzy number, and then

$$
\widetilde{A} \otimes \widetilde{B}= \begin{cases}\left(a^{(1)} b^{(1)}, a^{(2)} b^{(2)}, a^{(3)} b^{(3)}\right), & a^{(1)} \geq 0, \\ \left(a^{(1)} b^{(3)}, a^{(2)} b^{(2)}, a^{(3)} b^{(3)}\right), & a^{(1)}<0, a^{(3)} \geq 0, \\ \left(a^{(1)} b^{(3)}, a^{(2)} b^{(2)}, a^{(3)} b^{(1)}\right), & a^{(3)}<0 .\end{cases}
$$

2.3. Comparison Operations. Let $\widetilde{A}=\left(a^{(1)}, a^{(2)}, a^{(3)}\right)$ and $\widetilde{B}=\left(b^{(1)}, b^{(2)}, b^{(3)}\right)$ be two triangular fuzzy numbers, and then greater-than and less-than operations can be defined as follows [25]:

$$
\begin{aligned}
& \widetilde{A} \geq \widetilde{B} \Longleftrightarrow a^{(1)} \geq b^{(1)}, a^{(2)} \geq b^{(2)}, a^{(3)} \geq b^{(3)}, \\
& \widetilde{A} \leq \widetilde{B} \Longleftrightarrow a^{(1)} \leq b^{(1)}, a^{(2)} \leq b^{(2)}, a^{(3)} \leq b^{(3)} .
\end{aligned}
$$




\section{Presentation of the Problem}

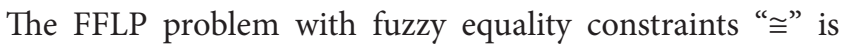
written as follows:

$$
\begin{array}{ll}
\operatorname{Max} & \widetilde{Z}=\widetilde{C} \otimes \widetilde{X}, \\
\text { s.t. } & \widetilde{A} \otimes \widetilde{X} \cong \widetilde{b},
\end{array}
$$

$\widetilde{X}$ is a nonnegative fuzzy vector,

where $\widetilde{C}=\left(\widetilde{c}_{j}\right)_{1 \times n}, \widetilde{X}=\left(\widetilde{x}_{j}\right)_{n \times 1}, \widetilde{A}=\left(\widetilde{a}_{i j}\right)_{m \times n}, \widetilde{b}=\left(\widetilde{b}_{i}\right)_{m \times 1}$,

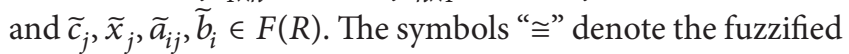
versions of " $=$ " and can be read as "approximately equal to."

Substituting $\widetilde{C}=\left(\widetilde{c}_{j}\right)_{1 \times n}, \widetilde{X}=\left(\widetilde{x}_{j}\right)_{n \times 1}, \widetilde{A}=\left(\widetilde{a}_{i j}\right)_{m \times n}, \widetilde{b}=$ $\left(\widetilde{b}_{i}\right)_{m \times 1},(\mathrm{P} 1)$ may be written as follows:

$\operatorname{Max} \widetilde{Z}=\sum_{j=1}^{n} \widetilde{c}_{j} \otimes \widetilde{x}_{j}$

s.t. $\quad \sum_{j=1}^{n} \widetilde{a}_{i j} \otimes \widetilde{x}_{j} \cong \widetilde{b}_{i}, \quad i=1,2, \ldots, m$,

$\tilde{x}_{j}$ is a nonnegative fuzzy number, $j=1,2, \ldots, n$.

Also, (P2) can be expressed as follows:

$$
\begin{array}{ll}
\operatorname{Max} & \widetilde{Z}=\sum_{j=1}^{n} \widetilde{c}_{j} \otimes \widetilde{x}_{j}, \\
\text { s.t. } & \sum_{j=1}^{n} \widetilde{a}_{i j} \otimes \widetilde{x}_{j} \leq \widetilde{b}_{i}, \quad i=1,2, \ldots, m, \\
& \sum_{j=1}^{n} \widetilde{a}_{i j} \otimes \widetilde{x}_{j} \gtrsim \widetilde{b}_{i}, \quad i=1,2, \ldots, m, \\
& \widetilde{x}_{j} \text { is a nonnegative fuzzy number, } \quad j=1,2, \ldots, n .
\end{array}
$$

Here the symbols " $\lesssim$ and $\gtrsim$ " denote the fuzzified versions of " $\leqslant$ and $\geqslant$ " and can be read as "approximately less/greater than or equal to."

As the decision maker (DM) knows that all the parameters and variables in each constraint of (P3) are fuzzy numbers, he may allow some violation of the right hand fuzzy number in each constraint. This violation can also be considered as a fuzzy number. Let $\widetilde{p}_{i}$ and $\widetilde{q}_{i}, i=$ $1,2, \ldots, m$, be fuzzy numbers determined by the DM giving his allowed maximum violation in the accomplishment of the $i$ th constraint and the $m+i$ th constraint of (P3), respectively. It means that the $\mathrm{DM}$ tolerates violations in each constraint of (P2) up the value $\widetilde{b}_{i}+\widetilde{p}_{i}$ and down the value $\widetilde{b}_{i}-\widetilde{q}_{i}, i=1,2, \ldots, m$, respectively. Based on these ideas, according to the resolution methods proposed in [4], (P3) will become

$$
\begin{array}{ll}
\operatorname{Max} & \widetilde{Z}=\sum_{j=1}^{n} \widetilde{c}_{j} \otimes \widetilde{x}_{j} \\
\text { s.t. } & \sum_{j=1}^{n} \widetilde{a}_{i j} \otimes \widetilde{x}_{j} \otimes \widetilde{b}_{i}+\widetilde{p}_{i}, \quad i=1,2, \ldots, m,
\end{array}
$$

$$
\sum_{j=1}^{n} \widetilde{a}_{i j} \otimes \widetilde{x}_{j} \otimes \widetilde{b}_{i}-\widetilde{q}_{i}, \quad i=1,2, \ldots, m,
$$

$\tilde{x}_{j}$ is a nonnegative fuzzy number, $\quad j=1,2, \ldots, n$,

where the symbols " $(₫)$ and $(\theta$ ) are relations between fuzzy numbers which preserve the ranking when fuzzy numbers are multiplied by positive scalars, and they can be anyone the DM chooses. Different kind of relation ( $)$ and $(€$ will lead to different models of CLP problems. In this paper, we assume that the relation $(\&$ and $(\nexists$ will be determined by using the comparison operations defined in Section 2.3.

Without any loss of generality, we assume that $\widetilde{c}_{j}$ and $\widetilde{a}_{i j}$ are nonnegative. Let $\widetilde{Z}=\left(Z^{(1)}, Z^{(2)}, Z^{(3)}\right), \widetilde{c}_{j}=\left(c_{j}^{(1)}, c_{j}^{(2)}, c_{j}^{(3)}\right)$, $\tilde{x}_{j}=\left(x_{j}^{(1)}, x_{j}^{(2)}, x_{j}^{(3)}\right), \widetilde{a}_{i j}=\left(a_{i j}^{(1)}, a_{i j}^{(2)}, a_{i j}^{(3)}\right), \widetilde{b}_{i}=\left(b_{i}^{(1)}, b_{i}^{(2)}, b_{i}^{(3)}\right)$, $\tilde{p}_{i}=\left(p_{i}^{(1)}, p_{i}^{(2)}, p_{i}^{(3)}\right), \widetilde{q}_{i}=\left(q_{i}^{(1)}, q_{i}^{(2)}, q_{i}^{(3)}\right)$, and then using the arithmetic operations between two triangular fuzzy numbers, (P4) may be written as

$$
\begin{gathered}
\operatorname{Max} \quad\left(Z^{(1)}, Z^{(2)}, Z^{(3)}\right) \\
=\sum_{j=1}^{n}\left(c_{j}^{(1)} x_{j}^{(1)}, c_{j}^{(2)} x_{j}^{(2)}, c_{j}^{(3)} x_{j}^{(3)}\right) \\
\text { s.t. } \quad \sum_{j=1}^{n}\left(a_{i j}^{(1)} x_{j}^{(1)}, a_{i j}^{(2)} x_{j}^{(2)}, a_{i j}^{(3)} x_{j}^{(3)}\right) \\
\ominus\left(b_{i}^{(1)}+p_{i}^{(1)}, b_{i}^{(2)}+p_{i}^{(2)}, b_{i}^{(3)}+p_{i}^{(3)}\right), \\
i=1,2, \ldots, m, \\
\sum_{j=1}^{n}\left(a_{i j}^{(1)} x_{j}^{(1)}, a_{i j}^{(2)} x_{j}^{(2)}, a_{i j}^{(3)} x_{j}^{(3)}\right) \\
\oslash\left(b_{i}^{(1)}-q_{i}^{(3)}, b_{i}^{(2)}-q_{i}^{(2)}, b_{i}^{(3)}-q_{i}^{(1)}\right), \\
i=1,2, \ldots, m, \\
\left(x_{j}^{(1)}, x_{j}^{(2)}, x_{j}^{(3)}\right) \text { is a nonnegative triangular } \\
\text { fuzzy number, } \quad j=1,2, \ldots, n .
\end{gathered}
$$

Using the comparison operations between two triangular fuzzy numbers, defined in Section 2.3, to deal with the 
inequality relation on the constraints, (P5) is converted into the following problem:

$\operatorname{Max}\left(Z^{(1)}, Z^{(2)}, Z^{(3)}\right)=\sum_{j=1}^{n}\left(c_{j}^{(1)} x_{j}^{(1)}, c_{j}^{(2)} x_{j}^{(2)}, c_{j}^{(3)} x_{j}^{(3)}\right)$

s.t. $\quad \sum_{j=1}^{n} a_{i j}^{(h)} x_{j}^{(h)} \leq b_{i}^{(h)}+p_{i}^{(h)}, \quad h=1,2,3, i=1,2, \ldots, m$, $\sum_{j=1}^{n} a_{i j}^{(h)} x_{j}^{(h)} \geq b_{i}^{(h)}-q_{i}^{(4-h)}, \quad h=1,2,3, i=1,2, \ldots, m$, $x_{j}^{(1)} \geq 0, \quad x_{j}^{(2)}-x_{j}^{(1)} \geq 0, \quad x_{j}^{(3)}-x_{j}^{(2)} \geq 0$, $j=1,2, \ldots, n$.

Generally, the DM usually knows little about the problem; moreover, constraints of (P3) have different tolerated violations for different $\mathrm{DM}$, so it is difficult for the DM to determine reasonable values of $p_{i}^{(h)}$ and $q_{i}^{(h)}, h=1,2,3$, $i=1,2, \ldots, m$. In this paper, the concept of similarity between two triangular fuzzy numbers is introduced to solve this problem. The key of this method is that the DM determines an allowed similarity level instead of allowed maximum tolerated violations in each constraint of (P3) by using the following inequalities:

$$
\begin{aligned}
& S\left(\widetilde{b}_{i}, \widetilde{b}_{i}+\widetilde{p}_{i}\right) \geq s, \quad i=1,2, \ldots, m, \\
& S\left(\widetilde{b}_{i}, \widetilde{b}_{i}-\widetilde{q}_{i}\right) \geq s, \quad i=1,2, \ldots, m, \\
& p_{i}^{(1)} \geq 0, \quad p_{i}^{(2)}-p_{i}^{(1)} \geq 0, \quad p_{i}^{(3)}-p_{i}^{(2)} \geq 0, \\
& i=1,2, \ldots, m \text {, } \\
& q_{i}^{(1)} \geq 0, \quad q_{i}^{(2)}-q_{i}^{(1)} \geq 0, \quad q_{i}^{(3)}-q_{i}^{(2)} \geq 0, \\
& i=1,2, \ldots, m \text {. }
\end{aligned}
$$

Here $S\left(\widetilde{b}_{i}, \widetilde{b}_{i}+\widetilde{p}_{i}\right)$ is the similarity between two triangular fuzzy numbers $\widetilde{b}_{i}$ and $b_{i}+\widetilde{p}_{i}, i=1,2, \ldots, m, S\left(\widetilde{b}_{i}, \widetilde{b}_{i}-\widetilde{q}_{i}\right)$ is the similarity between two triangular fuzzy numbers $\widetilde{b}_{i}$ and $\widetilde{b}_{i}-\widetilde{q}_{i}$, $i=1,2, \ldots, m$, and $s$ is the allowed similarity level given by the DM.

According to the definition of the similarity between two triangular fuzzy numbers, we have

$$
\begin{aligned}
& S\left(\widetilde{b}_{i}, \widetilde{b}_{i}+\widetilde{p}_{i}\right)=1-\frac{p_{i}^{(1)}+2 p_{i}^{(2)}+p_{i}^{(3)}}{4 u_{i}}, \quad i=1,2, \ldots, m, \\
& S\left(\widetilde{b}_{i}, \widetilde{b}_{i}-\widetilde{q}_{i}\right)=1-\frac{q_{i}^{(1)}+2 q_{i}^{(2)}+q_{i}^{(3)}}{4 u_{i}^{\prime}}, \quad i=1,2, \ldots, m,
\end{aligned}
$$

where $u_{i}=b_{i}^{(3)}+p_{i}^{(3)}-b_{i}^{(1)}, u_{i}^{\prime}=b_{i}^{(3)}+q_{i}^{(3)}-b_{i}^{(1)}$. In order to decrease the influence of variables $p_{i}^{(3)}$ and $q_{i}^{(3)}$ on $S\left(\widetilde{b}_{i}, \widetilde{b}_{i}+\widetilde{p}_{i}\right)$ and $S\left(\widetilde{b}_{i}, \tilde{b}_{i}-\widetilde{q}_{i}\right), i=1,2, \ldots, m$, respectively, we assume that $u_{i}=u_{i}^{\prime}=b_{i}^{(3)}-b_{i}^{(1)}$. Hence, from (6) and (10), as well as (7) and (11), we obtain the following inequalities, respectively:

$$
\begin{array}{r}
p_{i}^{(1)}+2 p_{i}^{(2)}+p_{i}^{(3)} \leq 4(1-s)\left(b_{i}^{(3)}-b_{i}^{(1)}\right), \\
i=1,2, \ldots, m, \\
q_{i}^{(1)}+2 q_{i}^{(2)}+q_{i}^{(3)} \leq 4(1-s)\left(b_{i}^{(3)}-b_{i}^{(1)}\right), \\
i=1,2, \ldots, m .
\end{array}
$$

Therefore, giving the DM allowed similarity level $s, \widetilde{p}_{i}$ and $\widetilde{q}_{i}$ can be determined by (8), (9), and (12). Adding those inequalities into constrains of (P6), we obtain the following problem:

$$
\begin{aligned}
& \operatorname{Max}\left(Z^{(1)}, Z^{(2)}, Z^{(3)}\right)=\sum_{j=1}^{n}\left(c_{j}^{(1)} x_{j}^{(1)}, c_{j}^{(2)} x_{j}^{(2)}, c_{j}^{(3)} x_{j}^{(3)}\right) \\
& \text { s.t. } \quad \sum_{j=1}^{n} a_{i j}^{(h)} x_{j}^{(h)} \\
& \leq b_{i}^{(h)}+p_{i}^{(h)}, \quad h=1,2,3, i=1,2, \ldots, m, \\
& \sum_{j=1}^{n} a_{i j}^{(h)} x_{j}^{(h)} \\
& \geq b_{i}^{(h)}-q_{i}^{(4-h)}, \quad h=1,2,3, i=1,2, \ldots, m, \\
& p_{i}^{(1)}+2 p_{i}^{(2)}+p_{i}^{(3)} \\
& \leq 4(1-s)\left(b_{i}^{(3)}-b_{i}^{(1)}\right), \quad i=1,2, \ldots, m, \\
& q_{i}^{(1)}+2 q_{i}^{(2)}+q_{i}^{(3)} \\
& \leq 4(1-s)\left(b_{i}^{(3)}-b_{i}^{(1)}\right), \quad i=1,2, \ldots, m, \\
& x_{j}^{(1)} \geq 0, \quad x_{j}^{(2)}-x_{j}^{(1)} \geq 0, \quad x_{j}^{(3)}-x_{j}^{(2)} \geq 0, \\
& j=1,2, \ldots, n, \\
& p_{i}^{(1)} \geq 0, \quad p_{i}^{(2)}-p_{i}^{(1)} \geq 0, \quad p_{i}^{(3)}-p_{i}^{(2)} \geq 0, \\
& i=1,2, \ldots, m \text {, } \\
& q_{i}^{(1)} \geq 0, \quad q_{i}^{(2)}-q_{i}^{(1)} \geq 0, \quad q_{i}^{(3)}-q_{i}^{(2)} \geq 0, \\
& i=1,2, \ldots, m \text {. }
\end{aligned}
$$

Now we have transformed the FFLP problem (P1) into (P7) using similarity measures. The main feature of (P7) is that the constraints are crisp linear by introducing allowed similarity level $s$. The definition of $s$-feasible solution about (P7) is given as follows.

Definition 5. Given an allowed similarity level $s$, for a decision matrix $X^{0}(s)=\left(x_{j}^{0^{(h)}}(s)\right)_{n \times 3}$, we will say that it is $s$-feasible solution of $(\mathrm{P} 7)$ if $X^{0}(s)$ satisfies constraints of (P7). 
According to transforming processes of (P1) to (P7), we may set the following proposition.

Proposition 6. A decision matrix $X^{0}(s)$ is s-fuzzy-feasible solution of $(P 1)$ if and only if $X^{0}(s)$ is s-feasible solution of $(P 7)$.

In the following, the set of the s-feasible solution of (P7) will be denoted by $\aleph(s)$, and it is evident that

$$
s_{1}<s_{2} \Longrightarrow \aleph\left(s_{1}\right) \supset \aleph\left(s_{2}\right) \text {. }
$$

Then, (P7) can be rewritten as

$$
\begin{aligned}
& \operatorname{Max} \quad\left(Z^{(1)}, Z^{(2)}, Z^{(3)}\right)=\sum_{j=1}^{n}\left(c_{j}^{(1)} x_{j}^{(1)}, c_{j}^{(2)} x_{j}^{(2)}, c_{j}^{(3)} x_{j}^{(3)}\right) \\
& \text { s.t. } \quad\left(\mathrm{x}_{j}^{(1)}, \mathrm{x}_{\mathrm{j}}^{(2)}, \mathrm{x}_{\mathrm{j}}^{(3)}\right) \in \aleph(\mathrm{s}), \quad \mathrm{j}=1,2, \ldots, \mathrm{n} .
\end{aligned}
$$

In order to transform the fuzzy objective into crisp one, we should consider expected value and uncertainty of fuzzy objective. We use a ranking function to define the expected value of fuzzy objective. Many ranking functions can be found in the literatures, and we choose the same ranking function, which is defined in Definition 4, used by Kumar et al. [20]. The uncertainty of fuzzy objective is measured using the difference between upper bound and lower bound of fuzzy objective value. Therefore, (P7) may be transformed into the following crisp problem:

$$
\begin{array}{ll}
\operatorname{Max} & \mathfrak{R}(\widetilde{Z})=\frac{1}{4} \sum_{j=1}^{n}\left(c_{j}^{(1)} x_{j}^{(1)}+2 c_{j}^{(2)} x_{j}^{(2)}+c_{j}^{(3)} x_{j}^{(3)}\right) \\
\operatorname{Min} & \Delta(\widetilde{Z})=\sum_{j=1}^{n} c_{j}^{(3)} x_{j}^{(3)}-\sum_{j=1}^{n} c_{j}^{(1)} x_{j}^{(1)} \\
\text { s.t. } & \left(x_{j}^{(1)}, x_{j}^{(2)}, x_{j}^{(3)}\right) \in \aleph(s), \quad j=1,2, \ldots, n .
\end{array}
$$

(P8) is an $s$-parametric crisp biobjective LP model. Giving the value of $s$, and we can solve the $s$-efficient solution, which is defined as follows.

Definition 7. Giving the value of $s, X^{*}(s) \in \aleph(s)$ is said to be $s$-efficient solution to the problem (P8) if there does not exist another $X^{0}(s) \in \aleph(s)$ such that

$$
\begin{gathered}
\Re\left(\widetilde{Z}\left(X^{0}(s)\right)\right) \geq \Re\left(\widetilde{Z}\left(X^{*}(s)\right)\right), \\
\Delta\left(\widetilde{Z}\left(X^{0}(s)\right)\right) \leq \Delta\left(\widetilde{Z}\left(X^{*}(s)\right)\right),
\end{gathered}
$$

where at least one of these inequalities is strict.

From Definition 7, we have the following proposition.

Proposition 8. All s-efficient solutions $X^{*}(s)$ to the problem $(P 8)$ are $s$-fuzzy-optimal solutions to the problem $(P 1)$ and reciprocally.

From Proposition 8, we can obtain the $s$-fuzzy-optimal solutions to the problem (P1) by solving (P8).

\section{The Auxiliary Three-Objective LP Model}

From (12), a bigger value of $s$ implies that the DM allows a smaller violation of the right hand fuzzy number in each constraint. So $s$ can be interpreted as the feasibility degree of constrains, the bigger the value of $s$ is; the higher the feasibility degree of constrains will be. However, from (13) and (P7), the bigger the value of $s$ is, the worst the objective value will be. Therefore, we want to find a balance solution between two goals: to improve the objectives function values and to increase the feasibility degree of constrains. According to the previous analysis, (P8) can be transformed into the following auxiliary three-objective LP model:

$$
\begin{aligned}
& \operatorname{Max} \mathfrak{R}(\widetilde{Z})=\frac{1}{4} \sum_{j=1}^{n}\left(c_{j}^{(1)} x_{j}^{(1)}+2 c_{j}^{(2)} x_{j}^{(2)}+c_{j}^{(3)} x_{j}^{(3)}\right), \\
& \operatorname{Min} \Delta(\widetilde{Z})=\sum_{j=1}^{n} c_{j}^{(3)} x_{j}^{(3)}-\sum_{j=1}^{n} c_{j}^{(1)} x_{j}^{(1)},
\end{aligned}
$$$$
\operatorname{Max} s
$$$$
\text { s.t. } \quad s_{m} \leq s \leq 1
$$$$
\left(x_{j}^{(1)}, x_{j}^{(2)}, x_{j}^{(3)}\right) \in \aleph(s), \quad j=1,2, \ldots, n,
$$

where $s_{m}$ is the allowed minimum similarity level, and it is specified by the DM according to his interests. The three objectives of (P9) represent the DM's preference for the alternative with the higher expected value, less uncertainty of objective, and the higher feasibility degree of constrains, respectively.

In the following, the set of the feasible solutions of (P9) will be denoted by $\aleph$.

Definition 9. $\left(X^{*}, s^{*}\right) \in \aleph$ is said to be an efficient solution to the problem (P9) if there does not exist another $\left(X^{0}, s^{0}\right) \in \mathcal{N}$ such that

$$
\begin{aligned}
\Re\left(\widetilde{Z}\left(X^{0}\right)\right) & \geq \Re\left(\widetilde{Z}\left(X^{*}\right)\right), \\
\Delta\left(\widetilde{Z}\left(X^{0}\right)\right) & \leq \Delta\left(\widetilde{Z}\left(X^{*}\right)\right), \\
s^{0} & \geq s^{*}
\end{aligned}
$$

where at least one of these inequalities is strict.

From Definitions 7 and 9 and Proposition 8, we have the following proposition.

Proposition 10. All efficient solutions $\left(X^{*}, s^{*}\right)$ to the problem (P9) are $s^{*}$-fuzzy-optimal solutions to the problem (P1) and reciprocally.

Proof. Let $\left(X^{*}, s^{*}\right)$ be an $s^{*}$-fuzzy-optimal solutions to the problem (P1) but not an efficient solution to the problem (P9), and then there exists another $\left(X^{0}, s^{0}\right) \in \aleph$ such that $\mathfrak{R}\left(\widetilde{Z}\left(X^{0}\right)\right) \geq \mathfrak{R}\left(\widetilde{Z}\left(X^{*}\right)\right), \Delta\left(\widetilde{Z}\left(X^{0}\right)\right) \leq \Delta\left(\widetilde{Z}\left(X^{*}\right)\right), s^{0} \geq s^{*}$, where at least one of these inequalities is strict. It implies, from Definition 7 , that $\left(X^{*}, s^{*}\right)$ is not an $s^{*}$-efficient solution to the problem (P8), and then according to Proposition 8, 
$\left(X^{*}, s^{*}\right)$ is not an $s^{*}$-fuzzy-optimal solutions to the problem (P1).

Reciprocally, let $\left(X^{*}, s^{*}\right)$ be an efficient solution to the problem (P9) but not an $s^{*}$-fuzzy-optimal solution to the problem (P1). Then from Proposition $8,\left(X^{*}, s^{*}\right)$ is not $s^{*}$ efficient solution to the problem (P8). So, from Definition 7, there exists another $\left(X^{0}, s^{*}\right) \in \aleph\left(s^{*}\right)$ such that $\mathfrak{R}\left(\widetilde{Z}\left(X^{0}\right)\right) \geq$ $\mathfrak{R}\left(\widetilde{Z}\left(X^{*}\right)\right), \Delta\left(\widetilde{Z}\left(X^{0}\right)\right) \leq \Delta\left(\widetilde{Z}\left(X^{*}\right)\right), s^{*}=s^{*}$, where at least one of these inequalities is strict. Therefore, $\left(X^{*}, s^{*}\right)$ is not an efficient solution to the problem (P9).

Now we have transformed the FFLP problem (P1) into (P9), which is a crisp three-objective LP problem. According to Proposition 10 , we can obtain the $s^{*}$-fuzzy-optimal solution of initial problem (P1) by solving (P9). In the following section, we will solve (P9) through CP approach.

\section{Compromise Solutions}

$\mathrm{CP}$ is a Multiple Criteria Decision Making approach which ranks alternatives according to their closeness to the ideal point. The best alternative is the one whose point is at the least distance from an ideal point in the set of efficient solutions [26].

In order to apply the $\mathrm{CP}$ approach to solve the problem (P9), we need to obtain the pay-off matrix. For this, we optimize each objective separately, calculating the values reached by the objectives on the optimal solution, respectively. Let $X_{T}^{*}, T=\mathfrak{R}, \Delta, s$, be the optimal solutions for each objective, and $\mathfrak{R}_{T}, \Delta_{T}, s_{T}$ the values reached by three objectives on the optimal solution $X_{T}^{*}, T=\mathfrak{R}, \Delta, s$, respectively. Then the obtained pay-off matrix can be expressed as in Table 1.

From Table 1, we know that the elements of principal diagonal $\left(\Re_{\mathfrak{R}}, \Delta_{\Delta}, s_{s}\right)$ form the ideal point. The anti-ideal point is $\left(\min \left\{\Re_{\Delta}, \Re_{s}\right\}, \max \left\{\Delta_{\mathfrak{R}}, \Delta_{s}\right\}, \min \left\{s_{\mathfrak{R}}, s_{\Delta}\right\}\right)$.

The distance between each objective value and the corresponding ideal point is

$$
\begin{gathered}
D_{1}=\Re_{\Re}-\mathfrak{R}(\widetilde{Z}), \quad D_{2}=\Delta(\widetilde{Z})-\Delta_{\Delta}, \\
D_{3}=s_{s}-s .
\end{gathered}
$$

As the objectives are measured with different units, it is necessary to homogenize the distances as

$$
\begin{gathered}
d_{1}=\frac{\mathfrak{R}_{\mathfrak{R}}-\mathfrak{R}(\widetilde{Z})}{\mathfrak{R}_{\mathfrak{R}}-\min \left\{\mathfrak{R}_{\Delta}, \mathfrak{R}_{s}\right\}}, \quad d_{2}=\frac{\Delta(\widetilde{Z})-\Delta_{\Delta}}{\max \left\{\Delta_{\mathfrak{R}}, \Delta_{s}\right\}-\Delta_{\Delta}}, \\
d_{3}=\frac{s_{s}-s}{s_{s}-\min \left\{s_{\mathfrak{R}}, s_{\Delta}\right\}} .
\end{gathered}
$$

The distance measure used in CP is the family of $L_{p^{-}}$ metrics given as

$$
L_{p}=\left[\sum_{k=1}^{3}\left(w_{k} d_{k}\right)^{p}\right]^{1 / p},
$$

where $w_{k}$ is the weight or relative importance attached to the $k$ th objective and $p$ is the topological metric that is a real number belonging to the closed interval $[1, \infty]$.
TABLE 1: The pay-off matrix.

\begin{tabular}{lccc}
\hline & $X_{\mathfrak{R}}^{*}$ & $X_{\Delta}^{*}$ & $X_{s}^{*}$ \\
\hline $\mathfrak{R}(\widetilde{Z})$ & $\mathfrak{R}_{\mathfrak{R}}$ & $\mathfrak{R}_{\Delta}$ & $\mathfrak{R}_{s}$ \\
$\Delta(\widetilde{Z})$ & $\Delta_{\mathfrak{R}}$ & $\Delta_{\Delta}$ & $\Delta_{s}$ \\
$s$ & $s_{\mathfrak{R}}$ & $s_{\Delta}$ & $s_{s}$ \\
\hline
\end{tabular}

A compromise solution is the one which minimizes $L_{p}$. Therefore, we have

$$
\begin{array}{ll}
\text { Min } & L_{p}=\left[\sum_{k=1}^{3}\left(w_{k} d_{k}\right)^{p}\right]^{1 / p} \\
\text { s.t. } & \left(\left(x_{j}^{(h)}\right)_{n \times 3}, s\right) \in \aleph .
\end{array}
$$

Obviously the solution of (P10) depends on the chosen metric. The most commonly obtained compromise solutions are for metrics $p=1$ and $p=\infty$ because for other metrics the nonlinear programming algorithms are needed [27]. Also, in the biobjective case they are the bounds of the whole compromise set $[28,29]$.

For $p=1$, the compromise solution closest to the ideal solution can be obtained by solving the following LP problem:

$$
\begin{array}{ll}
\text { Min } & L_{1}=\sum_{k=1}^{3}\left(w_{k} d_{k}\right) \\
\text { s.t. } & \left(\left(x_{j}^{(h)}\right)_{n \times 3}, s\right) \in \aleph .
\end{array}
$$

For $p=\infty$, the maximum divergence between individual discrepancies is minimized. Consequently, the compromise solution is obtained by solving the following problem:

$$
\begin{array}{ll}
\operatorname{Min} & L_{\infty}=\max _{k=1,2,3}\left\{w_{k} d_{k}\right\} \\
\text { s.t. } & \left(\left(x_{j}^{(h)}\right)_{n \times 3}, s\right) \in \aleph .
\end{array}
$$

The previous problem is a min-max problem. Let $d_{m}=$ $\max _{k=1,2,3}\left\{w_{k} d_{k}\right\}$, and then it is reformulated as

$$
\begin{array}{ll}
\text { Min } & L_{\infty}=d_{m} \\
\text { s.t. } & w_{k} d_{k} \leq d_{m}, \quad k=1,2,3, \\
& \left(\left(x_{j}^{(h)}\right)_{n \times 3}, s\right) \in \aleph .
\end{array}
$$

For $p=1$, Yu showed that the solution of $(\mathrm{P} 10-1)$ is always Pareto efficient [30]. For $p=\infty$, if (P10-3) exists as a unique optimal solution, then it is an efficient solution to the problem (P9) [31]. If the uniqueness is not satisfied, then the efficiency is not guaranteed for all solutions [31, 32]. In order to obtain an efficient solution several approaches have been proposed in the literature [31-35]. 
A composite form of $\mathrm{CP}$ for $p=1$ and $p=\infty$ can be obtained by minimizing a linear combination between $L_{1}$ and $L_{\infty}$; that is,

$$
\begin{array}{ll}
\text { Min } & L_{c}=(1-\lambda) d_{m}+\lambda \sum_{k=1}^{3}\left(w_{k} d_{k}\right) \\
\text { s.t. } & w_{k} d_{k} \leq d_{m}, \quad k=1,2,3, \\
& \left(\left(x_{j}^{(h)}\right)_{n \times 3}, s\right) \in \aleph,
\end{array}
$$

where $\lambda \in[0,1] . \lambda$ can be interpreted as a trade-off or marginal rate of substitution between $L_{1}$ and $L_{\infty}$. When $\lambda=$ 1 , problem (P10-4) gives the compromise solution for $p=1$, and for $\lambda=0,(\mathrm{P} 10-4)$ gives the compromise solution for $p=\infty$. For any set of positive weights and any $\lambda>0$, the solutions of problem (P10-4) are efficient [36].

\section{Numerical Examples}

In this section two numerical examples are given to illustrate the proposed model and method.

Example 1. Let us solve the following FFLP problem with fuzzy equality constraints, which is like Example 6.1 given in [20] with the main difference that here constraints are fuzzy equality, not crisp equality. Consider

$$
\begin{array}{ll}
\operatorname{Max} & \widetilde{Z}=(1,6,9) \otimes \tilde{x}_{1} \oplus(2,3,8) \otimes \tilde{x}_{2} \\
\text { s.t. } & (2,3,4) \otimes \widetilde{x}_{1} \oplus(1,2,3) \otimes \widetilde{x}_{2} \cong(6,16,30), \\
& (-1,1,2) \otimes \tilde{x}_{1} \oplus(1,3,4) \otimes \tilde{x}_{2} \cong(1,17,30), \\
& \tilde{x}_{1}, \tilde{x}_{2} \text { are nonnegative triangular fuzzy numbers. }
\end{array}
$$

Solution. Let $\tilde{x}_{1}=\left(x_{1}^{(1)}, x_{1}^{(2)}, x_{1}^{(3)}\right)$; and $\tilde{x}_{2}=\left(x_{2}^{(1)}, x_{2}^{(2)}, x_{2}^{(3)}\right)$ then the given FFLP problem (EP 1) may be written as

$\operatorname{Max} \quad \widetilde{Z}=\left(x_{1}^{(1)}+2 x_{2}^{(1)}, 6 x_{1}^{(2)}+3 x_{2}^{(2)}, 9 x_{1}^{(3)}+8 x_{2}^{(3)}\right)$

s.t. $\quad\left(2 x_{1}^{(1)}+x_{2}^{(1)}, 3 x_{1}^{(2)}+2 x_{2}^{(2)}, 4 x_{1}^{(3)}+3 x_{2}^{(3)}\right) \cong(6,16,30)$, $\left(-x_{1}^{(3)}+x_{2}^{(1)}, x_{1}^{(2)}+3 x_{2}^{(2)}, 2 x_{1}^{(3)}+4 x_{2}^{(3)}\right) \cong(1,17,30)$, $\left(x_{1}^{(1)}, x_{1}^{(2)}, x_{1}^{(3)}\right),\left(x_{2}^{(1)}, x_{2}^{(2)}, x_{2}^{(3)}\right)$ are nonnegative triangular fuzzy numbers.

Suppose that the allowed minimum similarity level specified by the DM is $s_{m}=0.9$. According to (P9), we will solve the following three-objective LP model:

$\operatorname{Max} \mathfrak{R}(\widetilde{Z})$

$\operatorname{Min} \Delta(\widetilde{Z})=9 x_{1}^{(3)}+8 x_{2}^{(3)}-x_{1}^{(1)}-2 x_{2}^{(1)}$,

$\operatorname{Max} s$

s.t. $\quad 0.9 \leq s \leq 1$

$$
\left(x_{j}^{(1)}, x_{j}^{(2)}, x_{j}^{(3)}\right) \in \aleph(s), \quad j=1,2,
$$

where $\aleph(s)$ is the set of the $s$-feasible solution of (P7), which is determined using the following constrain inequalities:

$$
\begin{gathered}
2 x_{1}^{(1)}+x_{2}^{(1)} \leq 6+p_{1}^{(1)}, \quad 3 x_{1}^{(2)}+2 x_{2}^{(2)} \leq 16+p_{1}^{(2)}, \\
4 x_{1}^{(3)}+3 x_{2}^{(3)} \leq 30+p_{1}^{(3)}, \quad-x_{1}^{(3)}+x_{2}^{(1)} \leq 1+p_{2}^{(1)}, \\
x_{1}^{(2)}+3 x_{2}^{(2)} \leq 17+p_{2}^{(2)}, \quad 2 x_{1}^{(3)}+4 x_{2}^{(3)} \leq 30+p_{2}^{(3)}, \\
2 x_{1}^{(1)}+x_{2}^{(1)} \geq 6-q_{1}^{(1)}, \quad 3 x_{1}^{(2)}+2 x_{2}^{(2)} \geq 16-q_{1}^{(2)}, \\
4 x_{1}^{(3)}+3 x_{2}^{(3)} \geq 30-q_{1}^{(3)}, \quad-x_{1}^{(3)}+x_{2}^{(1)} \geq 1-q_{2}^{(1)}, \\
x_{1}^{(2)}+3 x_{2}^{(2)} \geq 17-q_{2}^{(2)}, \quad 2 x_{1}^{(3)}+4 x_{2}^{(3)} \geq 30-q_{2}^{(3)}, \\
p_{1}^{(1)}+2 p_{1}^{(2)}+p_{1}^{(3)} \leq 96(1-s), \\
p_{2}^{(1)}+2 p_{2}^{(2)}+p_{2}^{(3)} \leq 116(1-s), \\
q_{i}^{(1)} \geq 0, \quad q_{i}^{(2)}-q_{i}^{(1)} \geq 0, \quad q_{i}^{(3)}-q_{i}^{(2)} \geq 0, \quad i=1,2 . \\
q_{1}^{(1)}+2 q_{1}^{(2)}+q_{1}^{(3)} \leq 96(1-s), \\
q_{j}^{(1)}+2 q_{2}^{(2)}+q_{2}^{(3)} \leq 116(1-s), \\
x_{j}^{(2)}-x_{j}^{(1)} \geq 0, \quad x_{j}^{(3)}-x_{j}^{(2)} \geq 0, \quad j=1,2, \\
p_{i}^{(1)} \geq 0, \quad p_{i}^{(3)}-p_{i}^{(2)} \geq 0, \quad i=1,2,
\end{gathered}
$$

In order to obtain the pay-off matrix, we optimize each objective separately, calculating the values reached by the objectives on the optimal solution, respectively. The obtained pay-off matrix is shown in Table 2.

From Table 2, we know that the elements of principal diagonal $(41.34,56.36,1.0)$ form the ideal point. The anti-ideal point is $(33.42,91.20,0.9)$. 
TABLE 2: The pay-off matrix of (EP 2).

\begin{tabular}{lccc}
\hline & $X_{\mathfrak{R}}^{*}$ & $X_{\Delta}^{*}$ & $X_{s}^{*}$ \\
\hline $\mathfrak{R}(\widetilde{Z})$ & 41.34 & 33.42 & 34.50 \\
$\Delta(\widetilde{Z})$ & 91.20 & 56.36 & 66.00 \\
$s$ & 0.9 & 0.9 & 1.0 \\
\hline
\end{tabular}

From (16) and (17), the homogenized distance between each objective value and the corresponding ideal point is

$$
\begin{gathered}
d_{1}=\frac{41.34-(1 / 4)\left(x_{1}^{(1)}+2 x_{2}^{(1)}+12 x_{1}^{(2)}+6 x_{2}^{(2)}+9 x_{1}^{(3)}+8 x_{2}^{(3)}\right)}{41.34-33.42}, \\
d_{2}=\frac{9 x_{1}^{(3)}+8 x_{2}^{(3)}-x_{1}^{(1)}-2 x_{2}^{(1)}-56.36}{91.20-56.36}, \\
d_{3}=\frac{1.0-s}{1.0-0.9} .
\end{gathered}
$$

The compromise solution can be obtained by solving (P10). Let $w_{1}=0.35, w_{2}=0.35, w_{3}=0.30$, and then we have the following.

(i) For $p=1$, the compromise solution closest to the ideal solution can be obtained by solving the following LP problem:

$$
\begin{array}{ll}
\text { Min } & L_{1}=0.35 d_{1}+0.35 d_{2}+0.30 d_{3} \\
\text { s.t. } & \left(\left(x_{j}^{(h)}\right)_{2 \times 3}, s\right) \in \aleph .
\end{array}
$$

The optimal solution of (EP 4) is $x_{1}^{(1)}=0.63, x_{1}^{(2)}=2.33$, $x_{1}^{(3)}=2.73, x_{2}^{(1)}=4.75, x_{2}^{(2)}=4.75, x_{2}^{(3)}=5.73, s^{*}=0.985$. According to Proposition 10, $s^{*}$-fuzzy-optimal solutions to the problem $(\mathrm{EP} 1)$ are $\tilde{x}_{1}^{*}(0.985)=(0.63,2.33,3.32)$, $\tilde{x}_{2}^{*}(0.985)=(4.75,4.75,5.73)$.

Put $\tilde{x}_{1}^{*}(0.985), \tilde{x}_{2}^{*}(0.985)$ in objectives of (EP 2) and (EP 3), and then we have $\widetilde{Z}^{*}=(10.12,28.20,75.73), \mathfrak{R}\left(\widetilde{Z}^{*}\right)=$ 35.56, $\Delta\left(\widetilde{Z}^{*}\right)=65.61$.

(ii) For $p=\infty$, the compromise solution is obtained by solving the following LP problem:

$$
\begin{array}{ll}
\text { Min } & L_{\infty}=d_{m} \\
\text { s.t. } & 0.35 d_{1} \leq d_{m}, \\
& 0.35 d_{2} \leq d_{m}, \\
& 0.30 d_{3} \leq d_{m}, \\
& \left(\left(x_{j}^{(h)}\right)_{2 \times 3}, s\right) \in \aleph .
\end{array}
$$

The optimal solution of (EP 5) is $x_{1}^{(1)}=0.87, x_{1}^{(2)}=2.66$, $x_{1}^{(3)}=3.00, x_{2}^{(1)}=4.25, x_{2}^{(2)}=4.25, x_{2}^{(3)}=7.00, s^{*}=0.959$. According to Proposition 10, $s^{*}$-fuzzy-optimal solutions to the problem $(\mathrm{EP} 1)$ are $\tilde{x}_{1}^{*}(0.959)=(0.87,2.66,3.00)$, $\tilde{x}_{2}^{*}(0.959)=(4.25,4.25,7.00)$.
Put $\tilde{x}_{1}^{*}(0.959), \tilde{x}_{2}^{*}(0.959)$ in objectives of (EP 2) and (EP 3), and then we have $\widetilde{Z}^{*}=(9.38,28.70,82.93), \mathfrak{R}\left(\widetilde{Z}^{*}\right)=$ 37.43, $\Delta\left(\widetilde{Z}^{*}\right)=73.55$.

(iii) For a composite form of CP for $p=1$ and $p=\infty$, the compromise solution is obtained by solving the following LP problem:

$$
\begin{array}{ll}
\text { Min } & L_{c}=(1-\lambda) d_{m}+\lambda\left(0.35 d_{1}+0.35 d_{2}+0.30 d_{3}\right) \\
\text { s.t. } & 0.35 d_{1} \leq d_{m}, \\
& 0.35 d_{2} \leq d_{m}, \\
& 0.30 d_{3} \leq d_{m}, \\
& \left(\left(x_{j}^{(h)}\right)_{2 \times 3}, s\right) \in \aleph .
\end{array}
$$

Assuming that $\lambda=0.5$, then optimal solution of (EP 6) is $x_{1}^{(1)}=0.73, x_{1}^{(2)}=2.76, x_{1}^{(3)}=2.76, x_{2}^{(1)}=4.54, x_{2}^{(2)}=$ 4.54, $x_{2}^{(3)}=6.77, s^{*}=0.957$. According to Proposition 10, $s^{*}$-fuzzy-optimal solutions to the problem (EP 1) are $\tilde{x}_{1}^{*}(0.957)=(0.73,2.76,2.76), \tilde{x}_{2}^{*}(0.957)=(4.54,4.54,6.77)$.

Put $\tilde{x}_{1}^{*}(0.957), \tilde{x}_{2}^{*}(0.957)$ in objectives of (EP 2) and (EP 3), and then we have $\widetilde{Z}^{*}=(9.81,30.19,79.04), \mathfrak{R}\left(\widetilde{Z}^{*}\right)=$ 37.31, $\Delta\left(\widetilde{Z}^{*}\right)=69.23$.

Given different values of $\lambda$, we can also obtain the $s^{*}$ fuzzy-optimal solutions to the problem (EP 1); see Table 3. Comparing these solutions with the ideal point, DM may choose an acceptable optimal solution.

Example 2. Let us solve the following FFLP problem with crisp equality constraints [20]:

$$
\begin{array}{ll}
\operatorname{Max} & \widetilde{Z}=(1,6,9) \otimes \tilde{x}_{1} \oplus(2,3,8) \otimes \tilde{x}_{2} \\
\text { s.t. } & (2,3,4) \otimes \tilde{x}_{1} \oplus(1,2,3) \otimes \tilde{x}_{2}=(6,16,30), \\
& (-1,1,2) \otimes \tilde{x}_{1} \oplus(1,3,4) \otimes \tilde{x}_{2}=(1,17,30), \\
& \widetilde{x}_{1}, \tilde{x}_{2} \text { are nonnegative triangular fuzzy numbers. }
\end{array}
$$

Solution. Let $\tilde{x}_{1}=\left(x_{1}^{(1)}, x_{1}^{(2)}, x_{1}^{(3)}\right)$ and $\tilde{x}_{2}=\left(x_{2}^{(1)}, x_{2}^{(2)}, x_{2}^{(3)}\right)$, and then the previous FFLP problem may be written as

$$
\begin{array}{ll}
\operatorname{Max} & \widetilde{Z}=\left(x_{1}^{(1)}+2 x_{2}^{(1)}, 6 x_{1}^{(2)}+3 x_{2}^{(2)}, 9 x_{1}^{(3)}+8 x_{2}^{(3)}\right) \\
\text { s.t. } & \left(2 x_{1}^{(1)}+x_{2}^{(1)}, 3 x_{1}^{(2)}+2 x_{2}^{(2)}, 4 x_{1}^{(3)}+3 x_{2}^{(3)}\right)=(6,16,30), \\
& \left(-x_{1}^{(3)}+x_{2}^{(1)}, x_{1}^{(2)}+3 x_{2}^{(2)}, 2 x_{1}^{(3)}+4 x_{2}^{(3)}\right)=(1,17,30), \\
& \left(x_{1}^{(1)}, x_{1}^{(2)}, x_{1}^{(3)}\right),\left(x_{2}^{(1)}, x_{2}^{(2)}, x_{2}^{(3)}\right) \text { are nonnegative }
\end{array}
$$$$
\text { triangular fuzzy numbers. }
$$

As the constraints are crisp equality, $s=1$. According to (P9), we can solve the following two-objective LP model 
TABLE 3: The $s^{*}$-fuzzy-optimal solutions with different value of $\lambda$.

\begin{tabular}{lcccccc}
\hline$\lambda$ & $s^{*}$ & $\widetilde{x}_{1}^{*}\left(s^{*}\right)$ & $\widetilde{x}_{2}^{*}\left(s^{*}\right)$ & $\widetilde{Z}^{*}$ & $\Re\left(\widetilde{Z}^{*}\right)$ \\
\hline 0 & 0.959 & $(0.87,2.66,3.00)$ & $(4.25,4.25,7.00)$ & $(9.38,28.70,82.93)$ & 37.43 \\
0.1 & 0.959 & $(0.90,2.82,2.82)$ & $(4.20,4.20,7.00)$ & $(9.30,29.49,81.36)$ & 37.41 \\
0.2 & 0.957 & $(0.73,2.76,2.76)$ & $(4.54,4.54,6.77)$ & $(9.81,30.19,79.04)$ & 37.31 \\
0.3 & 0.957 & $(0.73,2.76,2.76)$ & $(4.54,4.54,6.77)$ & $(9.81,30.19,79.04)$ & 37.31 & 69.55 \\
0.4 & 0.957 & $(0.73,2.76,2.76)$ & $(4.54,4.54,6.77)$ & $(9.81,30.19,79.04)$ & 69.23 \\
0.5 & 0.957 & $(0.73,2.76,2.76)$ & $(4.54,4.54,6.77)$ & $(9.81,30.19,79.04)$ & 37.31 \\
0.6 & 0.957 & $(0.73,2.76,2.76)$ & $(4.54,4.54,6.77)$ & $(9.81,30.19,79.04)$ & 37.31 \\
0.7 & 0.985 & $(0.63,2.33,3.32)$ & $(4.75,4.75,5.73)$ & $(10.12,28.20,75.73)$ & 35.56 \\
0.8 & 0.985 & $(0.63,2.33,3.32)$ & $(4.75,4.75,5.73)$ & $(10.12,28.20,75.73)$ & 35.56 \\
0.9 & 0.985 & $(0.63,2.33,3.32)$ & $(4.75,4.75,5.73)$ & $(10.12,28.20,75.73)$ & 69.23 \\
1 & 0.985 & $(0.63,2.33,3.32)$ & $(4.75,4.75,5.73)$ & $(10.12,28.20,75.73)$ & 69.23 \\
\hline
\end{tabular}

to obtain the fuzzy optimal solution of the previous FFLP problem:

$$
\begin{array}{ll}
\operatorname{Max} & \mathfrak{R}(\widetilde{Z}) \\
& =\frac{1}{4}\left(x_{1}^{(1)}+2 x_{2}^{(1)}+12 x_{1}^{(2)}+6 x_{2}^{(2)}+9 x_{1}^{(3)}+8 x_{2}^{(3)}\right), \\
& \\
\text { Min } \quad \Delta(\widetilde{Z})=9 x_{1}^{(3)}+8 x_{2}^{(3)}-x_{1}^{(1)}-2 x_{2}^{(1)} & \\
\text { s.t. } \quad 2 x_{1}^{(1)}+x_{2}^{(1)}=6, \quad 3 x_{1}^{(2)}+2 x_{2}^{(2)}=16, \\
& 4 x_{1}^{(3)}+3 x_{2}^{(3)}=30, \quad-x_{1}^{(3)}+x_{2}^{(1)}=1, \\
& x_{1}^{(2)}+3 x_{2}^{(2)}=17, \quad 2 x_{1}^{(3)}+4 x_{2}^{(3)}=30, \\
& x_{j}^{(1)} \geq 0, \quad x_{j}^{(2)}-x_{j}^{(1)} \geq 0, \quad x_{j}^{(3)}-x_{j}^{(2)} \geq 0,
\end{array}
$$

In order to obtain the pay-off matrix, we optimize each objective separately. As the optimal solution is same for two objective, we have obtained the optimal solution of previous two-objective LP model: $x_{1}^{(1)}=1, x_{1}^{(2)}=2, x_{1}^{(3)}=3, x_{2}^{(1)}=4$, $x_{2}^{(2)}=5, x_{2}^{(3)}=6 \cdot \mathfrak{R}\left(\widetilde{Z}^{*}\right)=34.5, \Delta\left(\widetilde{Z}^{*}\right)=66$. Therefore, the fuzzy optimal solution of the given FFLP problem is $\tilde{x}_{1}=$ $(1,2,3), \widetilde{x}_{2}=(4,5,6), \widetilde{Z}^{*}=(9,27,75)$.

From the analysis and solution processes of the numerical examples, we have summarized the following advantages.

(i) The auxiliary model (P9) to solve the FFLP problem (P1) is linear, so it is very ease to solve.

(ii) The FFLP problem with crisp equality constraints in [20] is a special case of the FFLP problems with fuzzy equality constraints $(s=1)$.

(iii) In order to solve the FFLP problems with fuzzy equality constraints, the DM only determines one parameter instead of $6 \mathrm{~m}$ parameters. Moreover, the DM can get different fuzzy optimal solution giving different similarity level.
The main disadvantage of proposed method is that the number of constraints is increased when the FFLP problems with fuzzy equality constraints are converted into crisp one.

\section{Conclusions}

In this paper, an FFLP problem with fuzzy equality constraints has been presented. Also, an approach has been given to solve it. We first transform the FFLP problem with fuzzy equality constraints into the crisp three-objective LP model by considering expected value and uncertainty of fuzzy objective and the feasibility degree of fuzzy constrains. Then we solve it using a CP approach. To illustrate the proposed method, two numerical examples are solved.

For different comparison relations of fuzzy number and different measure methods of similarity, an FFLP problem can be converted into different auxiliary problems. The study of those problems will be the aim of a forthcoming paper.

\section{Acknowledgments}

This work was partially supported by the National Natural Science Foundation of China (no. 71202140) and the Humanities and Social Sciences Project of Ministry of Education under Grants 10YJC630009 and 12YJCZH065.

\section{References}

[1] Y. Tan and Y. Long, "Option-game approach to analyze technology innovation investment under fuzzy environment," Journal of Applied Mathematics, vol. 2012, Article ID 830850, 9 pages, 2012.

[2] L. Zhang, X. Xu, and L. Tao, "Some similarity measures for triangular fuzzy number and their applications in multiple criteria group decision-making," Journal of Applied Mathematics, vol. 2013, Article ID 538261, 7 pages, 2013.

[3] H. Tsai and T. Chen, "A fuzzy nonlinear programming approach for optimizing the performance of a four-objective fluctuation smoothing rule in a wafer fabrication factory," Journal of Applied Mathematics, vol. 2013, Article ID 720607, 15 pages, 2013.

[4] M. Delgado, J. L. Verdegay, and M. A. Vila, "A general model for fuzzy linear programming," Fuzzy Sets and Systems, vol. 29, no. 1, pp. 21-29, 1989. 
[5] H. Rommelfanger, "Fuzzy linear programming and applications," European Journal of Operational Research, vol. 92, no. 3 , pp. 512-527, 1996.

[6] G. Zhang, Y.-H. Wu, M. Remias, and J. Lu, "Formulation of fuzzy linear programming problems as four-objective constrained optimization problems," Applied Mathematics and Computation, vol. 139, no. 2-3, pp. 383-399, 2003.

[7] N. van Hop, "Solving fuzzy (stochastic) linear programming problems using superiority and inferiority measures," Information Sciences, vol. 177, no. 9, pp. 1977-1991, 2007.

[8] M. Jiménez, M. Arenas, A. Bilbao, and M. V. Rodríguez, "Linear programming with fuzzy parameters: an interactive method resolution," European Journal of Operational Research, vol. 177, no. 3, pp. 1599-1609, 2007.

[9] H.-C. Wu, "Optimality conditions for linear programming problems with fuzzy coefficients," Computers \& Mathematics with Applications, vol. 55, no. 12, pp. 2807-2822, 2008.

[10] X. Liu, "Measuring the satisfaction of constraints in fuzzy linear programming," Fuzzy Sets and Systems, vol. 122, no. 2, pp. 263$275,2001$.

[11] J. Chiang, "Fuzzy linear programming based on statistical confidence interval and interval-valued fuzzy set," European Journal of Operational Research, vol. 129, no. 1, pp. 65-86, 2001.

[12] K. D. Jamison and W. A. Lodwick, "Fuzzy linear programming using a penalty method," Fuzzy Sets and Systems, vol. 119, no. 1, pp. 97-110, 2001.

[13] T. León and E. Vercher, "Solving a class of fuzzy linear programs by using semi-infinite programming techniques," Fuzzy Sets and Systems, vol. 146, no. 2, pp. 235-252, 2004.

[14] N. Mahdavi-Amiri and S. H. Nasseri, "Duality results and a dual simplex method for linear programming problems with trapezoidal fuzzy variables," Fuzzy Sets and Systems, vol. 158, no. 17, pp. 1961-1978, 2007.

[15] K. Ganesan and P. Veeramani, "Fuzzy linear programs with trapezoidal fuzzy numbers," Annals of Operations Research, vol. 143, no. 1, pp. 305-315, 2006.

[16] H. R. Maleki, M. Tata, and M. Mashinchi, "Linear programming with fuzzy variables," Fuzzy Sets and Systems, vol. 109, no. 1, pp. 21-33, 2000.

[17] S. M. Hashemi, M. Modarres, E. Nasrabadi, and M. M. Nasrabadi, "Fully fuzzified linear programming, solution and duality," Journal of Intelligent and Fuzzy Systems, vol. 17, no. 3, pp. 253-261, 2006.

[18] T. Allahviranloo, F. H. Lotfi, M. K. Kiasary, N. A. Kiani, and L. Alizadeh, "Solving fully fuzzy linear programming problem by the ranking function," Applied Mathematical Sciences, vol. 2, no. 1-4, pp. 19-32, 2008.

[19] F. H. Lotfi, T. Allahviranloo, M. A. Jondabeh, and L. Alizadeh, "Solving a full fuzzy linear programming using lexicography method and fuzzy approximate solution," Applied Mathematical Modelling, vol. 33, no. 7, pp. 3151-3156, 2009.

[20] A. Kumar, J. Kaur, and P. Singh, "A new method for solving fully fuzzy linear programming problems," Applied Mathematical Modelling, vol. 35, no. 2, pp. 817-823, 2011.

[21] X. Guo and D. Shang, "Fuzzy approximate solution of positive fully fuzzy linear matrix equations," Journal of Applied Mathematics, vol. 2013, Article ID 178209, 7 pages, 2013.

[22] A. Kaufmann and M. M. Gupta, Introduction to Fuzzy Arithmetic: Theory and Applications, Van Nostrand Reinhold, New York, NY, USA, 1985.

[23] H.-S. Lee, "Optimal consensus of fuzzy opinions under group decision making environment," Fuzzy Sets and Systems, vol. 132, no. 3, pp. 303-315, 2002.
[24] T.-S. Liou and M. J. J. Wang, "Ranking fuzzy numbers with integral value," Fuzzy Sets and Systems, vol. 50, no. 3, pp. 247255, 1992.

[25] S.-J. Chen and C.-L. Hwang, Fuzzy Multiple Attribute Decision Making: Methods and Applications, vol. 375 of Lecture Notes in Economics and Mathematical Systems, Springer, Berlin, Germany, 1992.

[26] M. Zeleny, Multiple Criteria Decision Making, McGraw-Hill, New York, NY, USA, 1982.

[27] M. Arenas Parra, A. Bilbao Terol, B. Pérez Gladish, and M. V. Rodríguez Uría, "Solving a multiobjective possibilistic problem through compromise programming," European Journal of Operational Research, vol. 164, no. 3, pp. 748-759, 2005.

[28] M. Freimer and P. L. Yu, "Some new results on compromise solutions for group decision problems," Management Science, vol. 22, no. 6, pp. 688-693, 1976.

[29] F. Blasco, E. Cuchillo-Ibáñez, M. A. Morón, and C. Romero, "On the monotonicity of the compromise set in multicriteria problems," Journal of Optimization Theory and Applications, vol. 102, no. 1, pp. 69-82, 1999.

[30] P. L. Yu, "A class of solutions for group decision problems," Management Science, vol. 19, no. 8, pp. 936-946, 1973.

[31] M. Jiménez and A. Bilbao, "Pareto-optimal solutions in fuzzy multi-objective linear programming," Fuzzy Sets and Systems, vol. 160, no. 18, pp. 2714-2721, 2009.

[32] B. Werners, "Interactive multiple objective programming subject to flexible constraints," European Journal of Operational Research, vol. 31, no. 3, pp. 342-349, 1987.

[33] E. S. Lee and R.-J. Li, "Fuzzy multiple objective programming and compromise programming with Pareto optimum," Fuzzy Sets and Systems, vol. 53, no. 3, pp. 275-288, 1993.

[34] C. Hu, Y. Shen, and S. Li, "An interactive satisficing method based on alternative tolerance for fuzzy multiple objective optimization," Applied Mathematical Modelling, vol. 33, no. 4, pp. 1886-1893, 2009.

[35] M. A. Yaghoobi and M. Tamiz, "A method for solving fuzzy goal programming problems based on MINMAX approach," European Journal of Operational Research, vol. 177, no. 3, pp. 1580-1590, 2007.

[36] F. J. André and C. Romero, "Computing compromise solutions: on the connections between compromise programming and composite programming," Applied Mathematics and Computation, vol. 195, no. 1, pp. 1-10, 2008. 


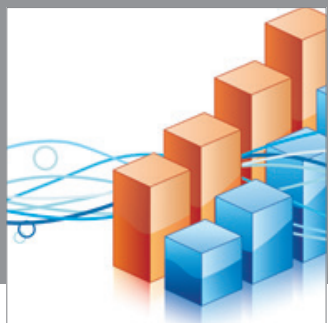

Advances in

Operations Research

mansans

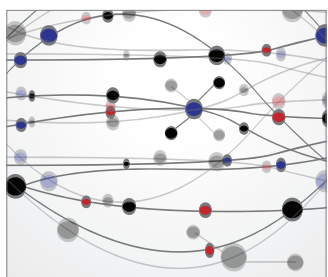

The Scientific World Journal
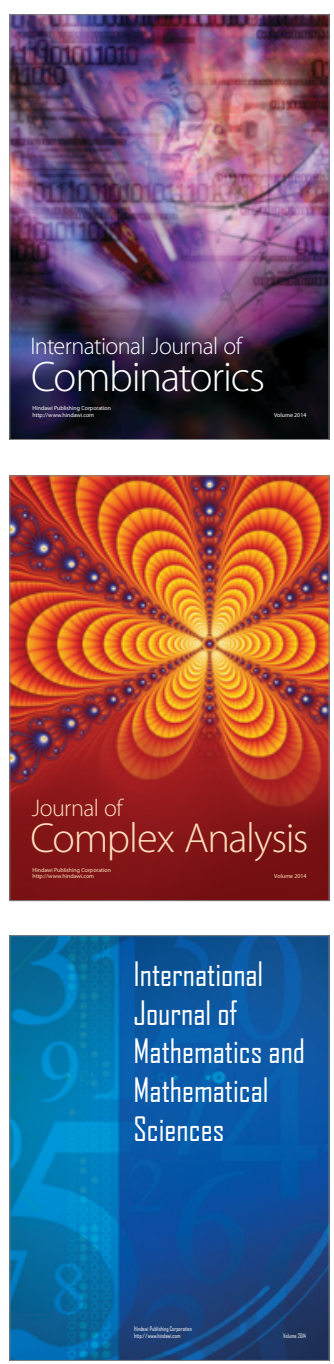
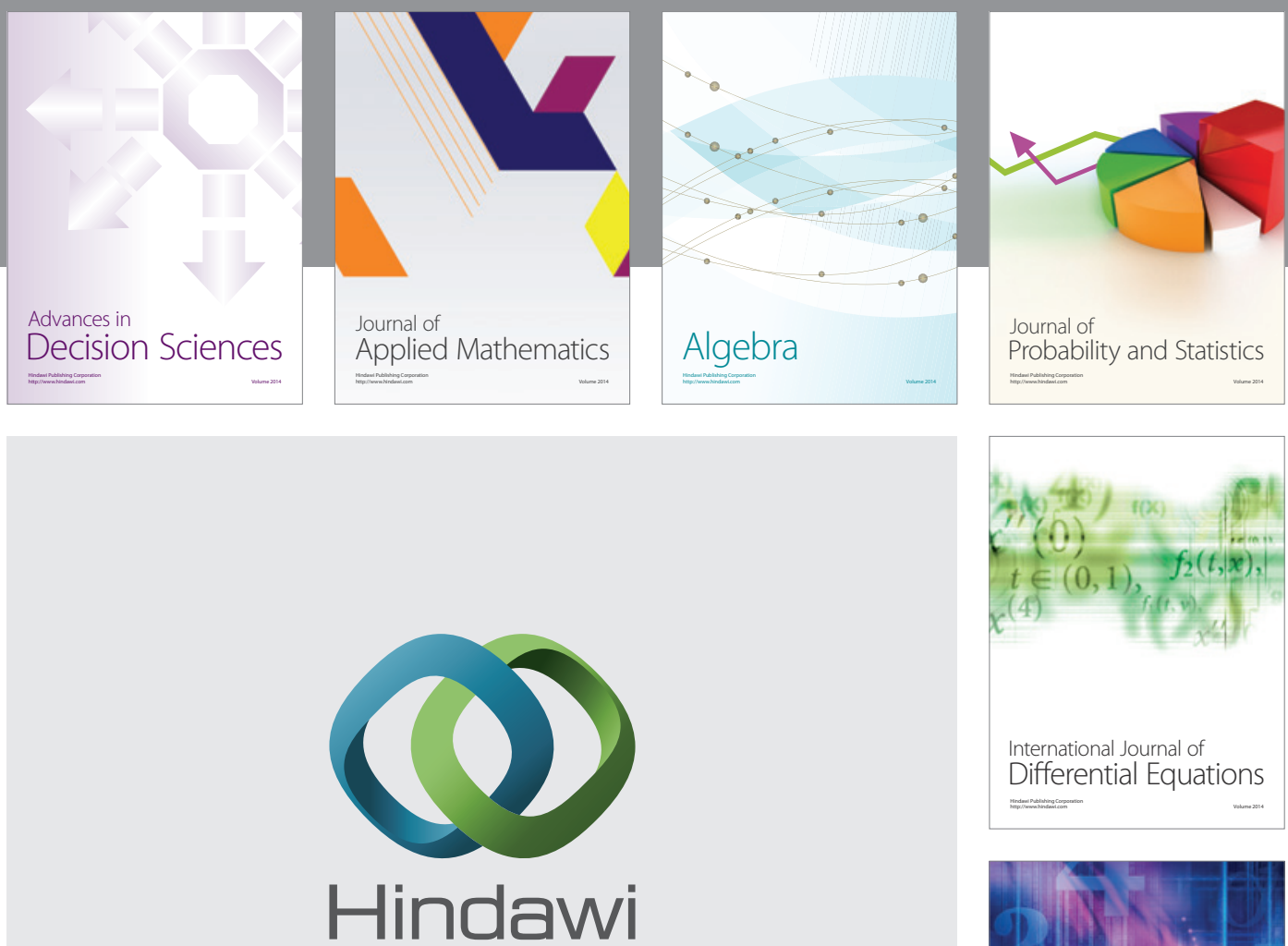

Submit your manuscripts at http://www.hindawi.com
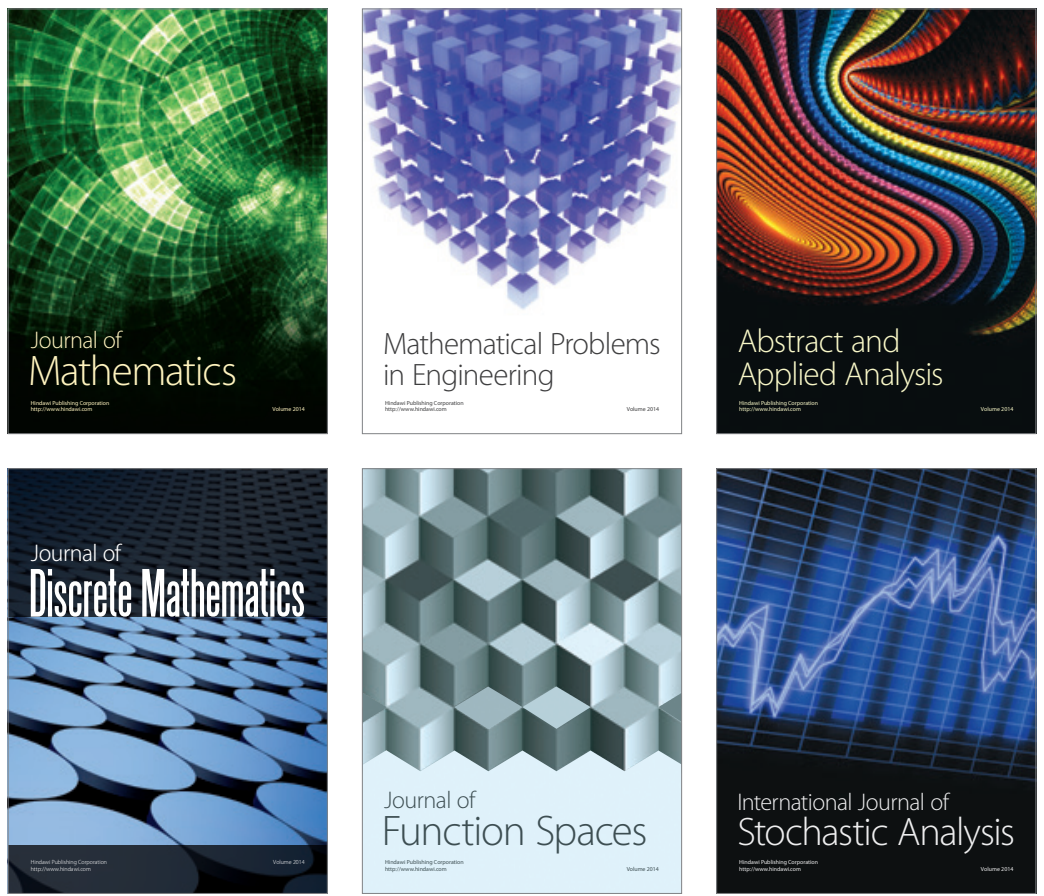

Journal of

Function Spaces

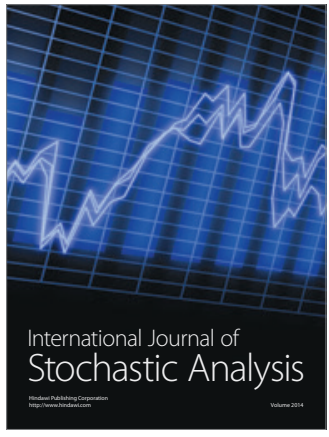

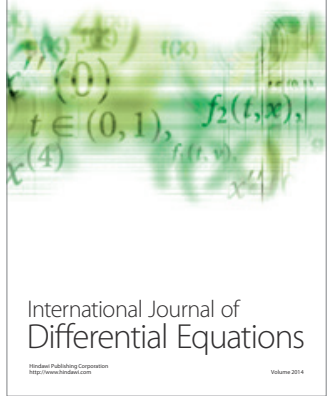
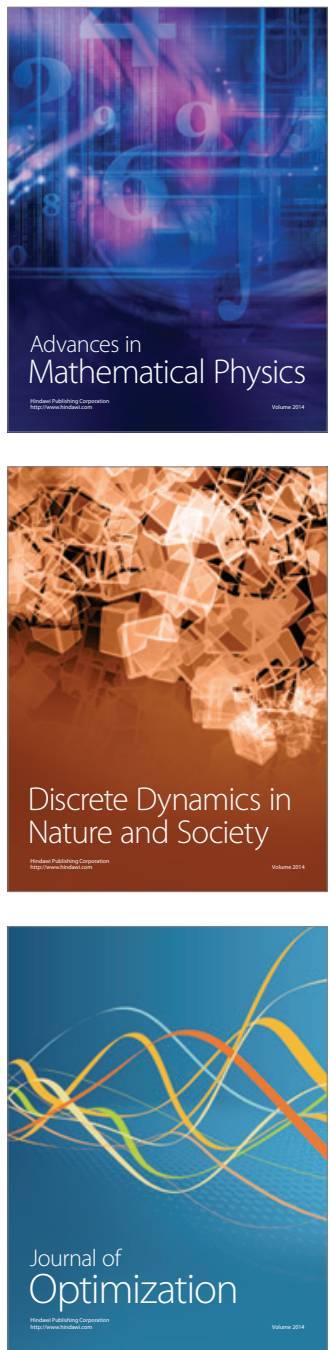